Revista Practicum, V1(1), julio-diciembre 2016

ISSN: $2530-4550$

\title{
EI Practicum y las prácticas externas en la formación universitaria
}

\section{Practicum and external practices in university education.}

Dr. Miguel A. Zabalza Beraza Universidad de Santiago de Compostela miguel.zabalza@usc.es

Zabalza, M.A. (2016). El Practicum y las prácticas externas en la formación universitaria. Revista Practicum, V1(1), 1-23 http://revistapracticum.com 


\section{Resumen}

Partiendo de la constatación de la progresiva importancia que ha ido adquiriendo el Prácticum en los Planes de estudio de las diferentes carreras, este trabajo analiza naturaleza y sentido desde tres perspectivas: (a) como componente curricular que se ha incorporado a la mayor parte de las propuestas formativas de las diferentes carreras; (b) como situación de aprendizaje en la que se espera que los estudiantes desarrollen conocimientos y competencias complementarios a los que adquieren en las aulas universitarias; (c) como experiencia personal con gran capacidad de movilizar no solo intelectual sino también emocional y afectivamente a nuestros estudiantes.

\section{Abstract}

Realizing the progressive importance that has been acquiring the Practicum in the Plans of study of the university careers, this paper analyzes its nature and meaning from three perspectives: (a) as a curricular component that has been incorporated into almost all careers; (b) as a learning situation in which students are expected to develop knowledge and skills that are complementary to those acquired in university classrooms; (c) as a personal experience with great capacity to mobilize students not only intellectually but also emotionally and affectively.

\section{Palabras clave}

Practicum, Prácticas externas, Planes de Estudio, Aprendizaje experiencial, Diseño curricular universitario.

\section{Key words}

Practicum, Outdoor training practices, Study plans, Experiential learning, University curriculum design. 


\section{ANTE PRIMA}

Me produce una gran satisfacción participar con un texto en la inauguración de este nuevo espacio de comunicación que es la Revista Practicum, editado por la Asociación REPPE. Hace muchos años que venimos acariciando esta idea. Los encuentros bianuales en el Monasterio de Poio para celebrar nuestros apreciados simposios sobre el Practicum nos parecían insuficientes para gestionar y visibilizar la gran producción de textos académicos e investigaciones que se van produciendo sobre el Practicum y las prácticas externas en las titulaciones universitarias. Afortunadamente, el grupo de personas que ha asumido la continuidad de los congresos de Poio ha logrado, también, poner en marcha la revista soñada. Ahora solamente nos falta desearles mucha suerte $y$ colaborar con ellos para que la revista se consolide y cumpla los exigentes objetivos que se ha propuesto.

\section{Introducción}

Para mí, hablar, escribir y reflexionar sobre el Practicum es una tarea habitual y gratificante. El Practicum y yo somos, a estas alturas, pareja de hecho. Llevamos más de 30 años cortejándonos. Cierto es que ambos nos hemos hechos mayores. $Y$ hemos ido cambiando de naturaleza y de estatus. Para bien, espero. El Practicum de joven era simplemente "prácticas" y jugaba un papelillo secundario en los estudios universitarios, salvo en algunas diplomaturas como magisterio o enfermería; ahora ya le llamamos Practicum y ha acabado ocupando un espacio relevante en los planes de estudio de la mayor parte de los grados y másteres. Yo también era más joven cuando comencé con estas cosas. Fue allá en los primeros ochenta cuando iniciamos nuestra participación en varios programas europeos (Petra y Leonardo da Vinci) estudiando los procesos de formación en alternacia en Alemania y el aprentisage con los colegas franceses. iQué tiempos aquellos! Después comenzamos con los Congresos de Poio en 1985 (Poio es un Monasterio precioso en la ría de Pontevedra donde cada dos años nos reunimos buena parte de los responsables y estudiosos del Practicum en España, Portugal, Italia y muchos que vienen de otros países, especialmente Sudamérica). Congresos estos de Poio que se han convertido en uno de los referentes nacionales, e incluso internacionales, de quienes trabajan sobre el Practicum en la Universidad. Y como guinda del 
pastel, hace un par de años traté de ir cerrando todo este ciclo en un libro sobre El Practicum y las prácticas en empresas en la formación universitaria1. Así que me siento en casa hablando de estas cosas.

En cualquier caso, el Practicum se ha convertido en un tema importante. Se habla y escribe mucho sobre él. Pero tiene el pecado original de que habiendo nacido muy vinculado a la práctica, al hacer, se habla más de experiencias que del fundamento y sentido curricular sobre el que dichas experiencias se basan. Tenemos más narrativas que teoría curricular. No es que yo pretenda llenar ese vacío, pero en esa línea se han movido mis trabajos. Estoy convencido de que el Practicum es un componente esencial del proceso formativo de nuestros estudiantes, pero también que el "ir de prácticas" es mucho más que dejar la universidad y los trabajos académicos por unas semanas para abrir un paréntesis de trabajo "indefinido y aleatorio" fuera de la universidad. Hemos pasado mucho tiempo esforzándonos porque todos nuestros estudiantes encontraran un lugar donde hacer las prácticas y organizando las condiciones materiales para que ese periodo se llevara a cabo sin contratiempos. Quizás haya llegado el tiempo en que, una vez superada esa fase inicial y necesaria, empecemos a preocuparnos más seriamente por la calidad $y$ pertinencia de las experiencias que nuestros estudiantes viven durante las prácticas, de las cosas que aprenden realmente en ellas. A ello pretendo dedicar este trabajo. Comenzaré situando el Practicum como experiencia formativa que se produce en un contexto institucional, la Universidad, y al socaire de los nuevos enfoques formativos a los que nuestras universidades se han ido adhiriendo. Continuaré analizando el sentido del Practicum desde una triple perspectiva: como componente de los planes de estudios de muchas carreras (¿qué papel juega en los diseños curriculares de las carreras?); como situación de aprendizaje (qué y cómo se aprende durante las prácticas) y como experiencia personal (cómo viven los estudiantes las prácticas?).

\footnotetext{
${ }^{1}$ Zabalza, M. (2013). El Practicum y las Prácticas en Empresas en la formación universitaria. A la búsqueda de una formación equilibrada. Madrid: Narcea.
} 


\section{El Practicum en la universidad}

La primera constatación que podemos hacer sobre el Practicum es que se trata de una actividad formativa que se desarrolla en la universidad. Por tanto, buena parte de su naturaleza y características se derivan del hecho de que es algo que se produce en un contexto particular, en este caso en la universidad como contexto de formación.

¿Cómo es la universidad que nos ha tocado vivir (a unos como profesores, a otros como estudiantes)? Pues habría que decir que se trata de una institución que lleva bastantes años luchando por sobrevivir, por seguir manteniendo su papel relevante en el mundo de la educación y la ciencia. Algunas personas tienen la impresión de que la universidad actual ha perdido protagonismo, que se ha vuelto marginal. Quizás sea cierto, pero de ninguna manera se puede decir que la Universidad actual resulta invisible a la sociedad, más bien, al contrario está sometida a un constante escrutinio que le obliga a ir con paso lento y a la defensiva, tratando de justificar todo lo que hace o propone, perdiendo autonomía al ritmo de las progresivas decisiones políticas que restringen su ámbitos de decisión y limitan sus recursos. Aquella vieja universidad que se refugiaba en los claustros, que se sentía libre del mundanal ruido para poder crear y pensar en libertad, se ha convertido en un nuevo agente social, se ha metido de lleno en la batalla científica, cultural, social y política. No solo se ha hecho visible sino que ha asumido el riesgo de concitar sobre sí expectativas y amenazas, demandas y desafíos. Se espera de ella que se convierta en motor del desarrollo científico y cultural de la sociedad a la que pertenece; en generadora de cohesión y desarrollo social a través de las herramientas de la cultura común y la profesionalización de las jóvenes generaciones. Pero para ello tiene que renovarse. Mucho.

En el contexto europeo a todo este proceso se le vinculó a la creación de la Europa del conocimiento. Una Europa que fuera más allá de la Europa del mercado común. No está resultando un proceso fácil como hemos podido comprobar en estos años pasados, llenos de debates e incertidumbres (titulaciones, créditos, competencias, indicadores de logro, acreditaciones, etc.). Uno de esos nuevos estribillos académicos ha sido el de la necesidad de fortalecer el sentido "profesionalizante" de las carreras. Y ahí aparece el Practicum 
como un elemento curricular central. Esa búsqueda de la empleabilidad como objetivo central de la formación ha supuesto un fuerte impulso en la potenciación de los periodos de prácticas en empresas e instituciones ajenas a la universidad pero que se han vinculado a ella bajo diferentes tipos de convenios de colaboración. Así, el Practicum y/o las prácticas externas son hoy mucho más fuertes de lo que lo eran en los años 90 y como toda iniciativa educativa que crece rápidamente precisa de momentos de reflexión y de fijación de su sentido curricular para evitar que se convierta en una pieza suelta del sistema formativo y deje de cumplir la función que tiene encomendada. Sobre eso va este texto.

Cuando hablamos de Practicum estamos integrando en esa categoría todo un conjunto de actuaciones curriculares con contenidos y sentido diferentes y a las que se ha dado, en función de las diversas tradiciones institucionales, nombres diferentes. Al final, en cualquier caso, se trata de periodos de formación que, aunque integrados en sus planes de estudios, los estudiantes realizan fuera de la institución académica. Cerrado ya el proceso de Bolonia podría decirse que, al menos en el contexto español, prácticamente todas las carreras cuentan con periodos de Practicum. Y como suele acontecer en este tipo de procesos, la fase inicial de atención a los aspectos administrativos y organizacionales (muy diferentes a los aplicados a los otros momentos más académicos de la formación) ha dado paso a consideraciones más cualitativas. No basta con contar con prácticas externas, es necesario que esas prácticas sean de calidad y cumplan los objetivos formativos que se les atribuyen. Tres aspectos podríamos destacar:

1. El Practicum existe y, de hecho, se ha generalizado a la mayor parte de las carreras universitarias. Los estudiantes pasan parte de su tiempo en empresas e instituciones donde conocen in situ el trabajo de los profesionales de su ramo y participan, en la medida en que se lo permiten, en las actividades que allí se desarrollan.

2. En algunas carreras la relevancia curricular de ese periodo de prácticas externas es muy alta, llegando a constituir en torno al $30 \%$ del periodo formativo. Requiere, por tanto, de una fuerte atención a sus características y ha de ser planificado de forma tal que resulte un tiempo valioso y eficaz en la formación de los estudiantes. Ello exige un notable empeño a gestores y docentes universitarios. Pero también a los estudiantes. 
3. Es así que la cuestión de la calidad y de las "buenas prácticas en el PRATICUM" se convierte en ese contexto en una consideración ineludible (Shulman, 1986²; Zabalza, 2012³). Pero, a su vez, es algo que aún no se ha hecho. No disponemos de mecanismos ni instrumentos para evaluar el Practicum que está ahí como una especie de missing point en los actuales sistemas de evaluación de la calidad de las titulaciones. Lo he demandado a las Agencias de Calidad, en las que yo mismo he estado, pero, que yo sepa, poco se ha avanzado en ese aspecto.

Podríamos decir que estamos, por tanto, ante una profunda innovación curricular que supone importantes modificaciones en la forma de pensar la universidad y la formación que los estudiantes reciben en ella:

a) Se renuncia al sentido excluyente de la universidad como contexto formativo que actuaba cerrado sobre sí mismo y sobre su propia lógica formativa. Nuevos agentes formativos entran a formar parte del plan de formación: tanto la institución académica como el profesorado están llamados a compartir la función formativa con otras instituciones y otros profesionales.

b) Se enriquecen los escenarios de formación y sus contenidos. Los estudiantes se forman a través de las disciplinas académicas y las coreografías didácticas propias de la universidad pero también a través de la participación en actividades de tipo profesional supervisadas por profesionales en ejercicio.

c) Se diversifica el estatus de los estudiantes y los compromisos que asumen. Durante sus periodos de prácticas desarrollarán tareas y asumirán responsabilidades diferentes a las que asumen como alumnos. Deben atender tanto a los empleadores que los integran en sus organizaciones como a los sujetos con los que entran en relación como estudiantes en prácticas.

2 Shulman, L.S. (1986). Paradigms and Research Programs in the Study of Teaching: a contemporary perspective, en M.C. Wittrock (Dir.), Handbook of Research on Teaching, ( $3^{a}$ ed.) (pp. 3-36), N. York: McMillan.

3 Zabalza, M.A. (2012). El estudio de las buenas prácticas docentes" en la enseñanza universitaria. REDU Revista de Docencia Universitaria, 10(1), 17-40. 
d) Se amplia y enriquece el contexto de aprendizaje. Ya no se trata solo de aprender a través las explicaciones del profesor, de los libros o las actividades de laboratorio programadas en sede académica. El espectro de las experiencias de aprendizaje se amplía a los contextos reales de trabajo. $Y$ en ese doble juego de referentes teóricos y prácticos se facilita la comprensión y el dominio de los contenidos a aprender.

Entramos con ello en el ámbito más estricto de la formación y, en sentido más profundo, en el ámbito de la calidad de la formación. El Practicum constituye no una mera aproximación a los contextos profesionales para que los estudiantes se sientan más motivados por la carrera que estudian, sino una nueva manera de afrontar su formación diversificando los escenarios, ampliando el espectro de agentes formativos y enriqueciendo las competencias y significados de las cosas que van aprendiendo. El buen Practicum permite a los estudiantes tomar contacto con la realidad para la que se preparan como futuros profesionales, pero ha de posibilitar, a su vez, que esa experiencia sea rica formativamente y cumpla sus objetivos de cara al aprendizaje. Desafortunadamente no siempre hacemos las cosas bien en ese sentido.

En realidad, tenemos que reconocer que nuestras instituciones académicas tienen más tradición y más know how en organizar la docencia clásica que en organizar el Practicum. $Y$ eso se nota. Tampoco es una tarea fácil. El Practicum posee características diferenciales bastante notables en relación al resto de los componentes del proceso formativo universitario. Como pieza novedosa en la estructura curricular de las titulaciones, no siempre ha acabado por integrarse adecuadamente en la malla de disciplinas de nuestros Planes de estudios. Todavía figura como un componente excesivamente aislado y desconectado del resto de los componentes formativos (las clases, los seminarios, las prácticas de laboratorio, los exámenes, etc.). En algunos casos, ni siquiera ha adquirido carta de identidad curricular y aparece como algo extracurricular y opcional al que solo unos pocos estudiantes pueden acceder. Tampoco es infrecuente que sus propósitos se alejen del sentido formativo de las carreras y tengan más de función socializadora (acostumbrarse a trabajar en una empresa o institución) y/o de facilitación de empleo 
(mejorar las posibilidades de ser contratado en aquellas empresas en las que se hacen las prácticas).

Es justamente en este marco de condiciones institucionales y curriculares donde debemos situar el tema de la formación: para qué sirve el Practicum, qué se supone que ha de aportar a nuestros estudiantes. Podría pensarse que eso tiene una fácil respuesta: la condición básica del Practicum es que se trata de un momento curricular que complementa la formación académica que se recibe en las aulas. Pero no es tan sencillo o eso parece si se analizan las propuestas de prácticas que se hacen en las diferentes titulaciones.

Para resumir este primer asunto, tres consideraciones podemos extraer de los párrafos anteriores:

-El Practicum constituye una parte importante de la carrera que requiere procesos de planificación y seguimiento específicos, al igual que el resto de los componentes curriculares.

-Siendo que se trata de algo muy diferente a las materias convencionales, requiere de actuaciones curriculares (selección, planificación, desarrollo, seguimiento, evaluación, acreditación) también diferenciadas. El hecho de que en estos periodos de prácticas van a intervenir instituciones y agentes formativos distintos a los académicos complica el proceso y obliga a modalidades de planificación y seguimiento más cuidados si cabe.

-Aunque ciertas experiencias de prácticas externas han sido planificadas con sentido en sí mismas (como unidades curriculares autónomas y gestionadas por departamentos especializados y profesorado ad hoc), su sentido como Practicum curricular altera ese estatus independiente y las vincula al conjunto de las disciplinas y experiencias formativas que los estudiantes han de desarrollar durante su carrera. No actúan como experiencias independientes sino complementarias del resto de actuaciones formativas, cuyos objetivos deben compartir, cuyos contenidos deben ayudar a comprender mejor, cuyas competencias deben consolidar en otros contextos diferentes a los académicos. En realidad, el Practicum resulta un recurso curricular cuya principal aportación reside en generar un contexto que permite una mayor integración entre 
teoría y práctica, entre aprendizajes disciplinares y aplicación del conocimiento en contextos profesionales reales.

Desde esta perspectiva curricular, los mismos propósitos formativos (en términos de objetivos, aprendizajes y competencias) que se atribuyen a las diversas disciplinas del currículo habrán de proyectarse, adaptadas, a las diferentes situaciones en que los/las estudiantes realizan su Practicum.

\section{El Practicum como elemento clave de la propuesta formativa universitaria}

Tomando en consideración todo lo hasta aquí señalado, el análisis del compromiso formativo del Practicum (las exigencias que plantea un buen Practicum, capaz de cumplir la función formativa que se le encomienda) nos plantea la necesidad de abordarlo desde una triple perspectiva: como componente curricular; como momento de aprendizajes; como oportunidad de desarrollo personal.

\subsection{El Practicum como componente curricular.}

Considerar las prácticas externas de las prácticas bajo el prisma de su posición y función en el currículo formativo de las diversas Facultades o Escuelas Técnicas requiere analizar en qué medida cumple las siguientes condiciones:

a) Un contexto de convenios y acuerdos con empresas e instituciones relevantes donde los estudiantes puedan desarrollar unas prácticas eficaces. Esos convenios deberían establecer con claridad los compromisos que cada institución asume.

b) Una buena integración en el proyecto global de la titulación (en el Plan de estudios).

c) Una buena estructura interna como documento curricular (incluyendo un modelo de aprendizaje coherente con el que postula la propia titulación).

d) Recursos materiales y personales puestos a disposición del desarrollo del plan de prácticas

Obviamente, el punto de partida es que el Practicum, al igual que el resto de los componentes que configuran una carrera universitaria, tiene como propósito básico la formación personal y profesional de los estudiantes. Su capacidad de impacto formativo en los estudiantes va a depender de que el contexto en el que se realice ofrezca buenas 


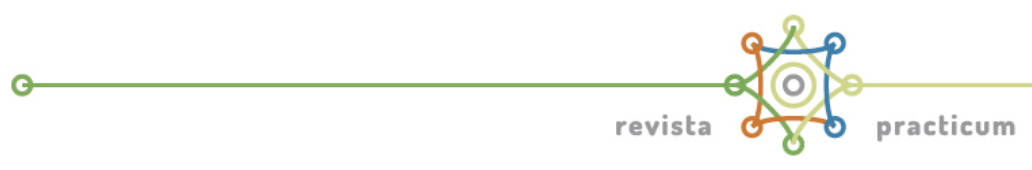

oportunidades de aprendizaje y de que su propia estructura interna como proceso formativo resulte adecuada. Por tanto, como pieza curricular, el Practicum debe poseer la condición de coherencia (que sus propósitos y el tipo de acciones que incluya estén bien alineadas con el proyecto formativo que la carrera plantea), la condición de centralidad (que tales propósitos y acciones resulten importantes para la profesión) y la condición de complementariedad (que el periodo de prácticas constituya un todo integrado con el conjunto de actividades de la carrera, de forma que se refuercen mutuamente).

No podremos desarrollar buenas prácticas si no contamos con centros de prácticas capaces de responder a los propósitos formativos que se les plantean (por ejemplo, si no realizan tareas pertinentes a la carrera de nuestros estudiantes, si no poseen la tecnología requerida, si no tienen experiencia en tareas formativas, si no buscan la formación de nuestros estudiantes sino su aprovechamiento como mano de obra barata). Por ese motivo es tan importante que los acuerdos interinstitucionales dejen claros los compromisos que las instituciones participantes asumen y las contraprestaciones que entre universidad y centros de prácticas se establecen.

Tampoco lograremos desarrollar un buen Practicum si el proyecto que lo desarrolla se aleja de las características propias de la planificación didáctica: una buena contextualización; una explicitación de los propósitos formativos que incluyan el conjunto de aprendizajes y competencias que le corresponde desarrollar; los nuevos conocimientos conceptuales y operativos que se espera obtener durante las prácticas; las actividades o experiencias que se desarrollarán durante el periodo de prácticas; los sistemas de supervisión que acompañarán las prácticas; la forma en que serán evaluados los resultados de aprendizaje y el propio programa de prácticas.

Con frecuencia, el periodo de prácticas se desgaja en exceso del conjunto de las disciplinas y otras acciones formativas que incluye una carrera universitaria. La tendencia a la atomización de las acciones formativas y a la segmentación de las materias y asignaturas como unidades independientes (los modelos curriculares por yuxtaposición donde cada disciplina o unidad curricular sigue un proceso separado y sin apenas relaciones las demás del Plan de 
Estudios) afecta gravemente a la articulación curricular y a la necesaria complementación entre las unidades curriculares.

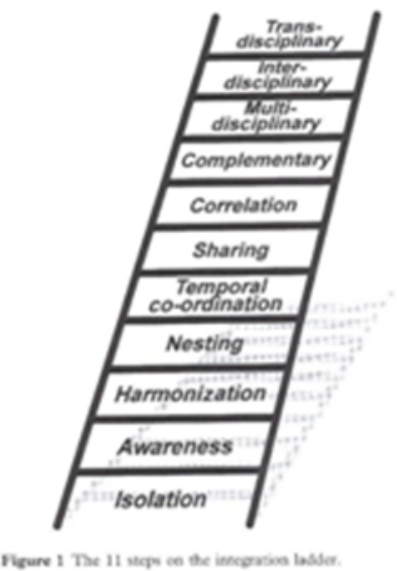

Ilustración 1, Escalera de Harden, extraída de Harden,2000

La conocida escalera de Harden $(2000)^{4}$ marca los 11 peldaños que separan una estructura de unidades curriculares separadas (isolation) de un planteamiento transdisciplinar 0 modular. Normalmente estamos funcionando en el primero de esos peldaños: las materias aisladas y desconectadas. Un Practicum funcionando aisladamente (como una disciplina desligada del resto y con su propio profesorado) tiene pocas posibilidades de cumplir con su función de integración entre teoría académica y práctica profesional. Por el contrario, desarrolla bien su función cuando forma parte de los diferentes clusters de materias que forman parte de una titulación organizada como un proyecto formativo bien integrado. En ese sentido los currículos modulares o los organizados por competencias (los situados en la parte alta de la escala de Harden) son aquellos en los que el Practicum encuentra mejor acomodo y ofrece resultados formativos más interesantes.

Ni qué decir tiene que el Practicum constituye un componente curricular que requiere de recursos apropiados. Dado que se trata de una acción formativa out-door y claramente diferenciada de las clases y sesiones de laboratorio que se realizan en sede universitaria, el profesorado que se dedique a tutorizar el Practicum (mejor si se combina esa tutoría con la impartición de otras materias para evitar el aislamiento curricular al que nos hemos referido) debe tener

\footnotetext{
${ }^{4}$ Harden, R.M. (2000). The integration ladder: a tool for curriculum planning and evaluation. Medical Education, 34(7), 551-557
} 
alguna experiencia en las tareas profesionales de la carrera y conocimiento de la dinámica de trabajo en los contextos profesionales a los que acudan los estudiantes en prácticas. Otros recursos materiales e informáticos serán necesarios para facilitar la movilidad de nuestros estudiantes, para gestionar el proceso y tener un seguimiento constante de la situación de los estudiantes e, incluso, para poder contar con algún tipo de contraprestaciones a los profesionales que se van a encargar de atender y completar la formación de los alumnos. Resulta difícil ser exigente con las prácticas si éstas están pobremente dotadas, si se les hace depender exclusivamente de la buena voluntad y la disponibilidad solidaria de las personas que los acogen.

En definitiva, la dimensión curricular del Practicum debe garantizar que la propuesta de prácticas externas que hagamos a nuestros estudiantes esté bien integrada en el proyecto formativo que les ofrece la Facultad en la que se forman. No se trata de enviarlos fuera de la universidad a cualquier sitio para que se involucren en las actividades que vayan surgiendo, sino de hacerlo con un plan bien diseñado de formación, en consonancia con las diversas fases de su progreso en la carrera universitaria que cursan.

\subsection{El Practicum como situación de aprendizaje.}

Aunque, en algunos casos, se intenta justificar las prácticas por su importancia como transición al empleo, no es ése su auténtico sentido, al menos si se analizan bajo la perspectiva del Practicum. El objetivo de las prácticas es completar los aprendizajes y la formación que se obtiene en la universidad. Y no está de más recordar que esta orientación al aprendizaje es una de las líneas básicas de actuación en la filosofía de Bolonia. Lo importante no es tener un programa vistoso o una oferta de posibilidades amplia y variada. Al final, lo que importa es lo que los estudiantes aprenden durante el proceso. Eso es lo que va a legitimar la aparición del Practicum en nuestras titulaciones y lo que constituirá el criterio clave para su valoración como buena práctica: que los alumnos y alumnas que lo realicen obtengan aprendizajes relevantes para su formación. Y eso depende, entre otras cosas, de varios aspectos que podríamos considerar en este apartado:

a) la organización interna del proceso de prácticas que se ofrezca a los estudiantes 
b) el tipo de actividades $y / 0$ de compromisos que se les soliciten o encomienden

c) el tipo de supervisión que se establezca

d) el vigor y profundidad de la experiencia en relación al contexto profesional en el que desarrollen su Practicum

e) el propio diseño de la coreografía de aprendizaje que se haya planteado.

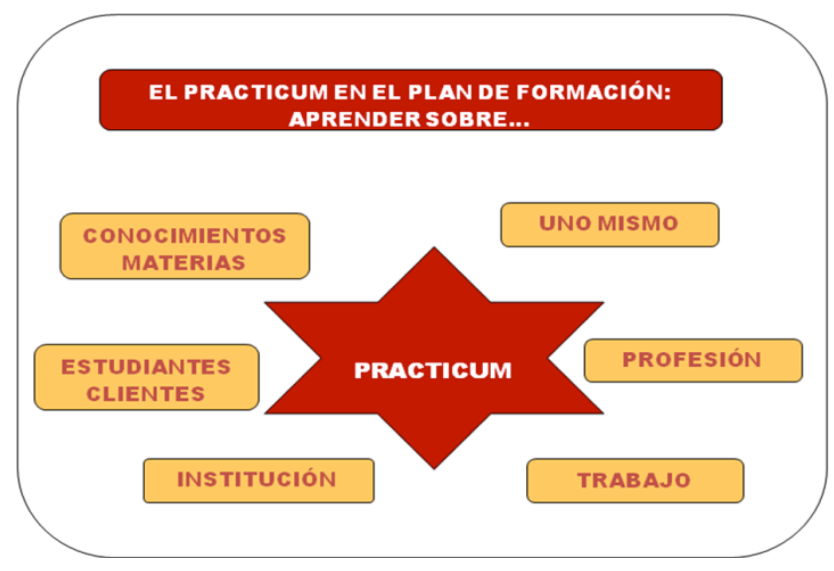

Ilustración 2, Aprendizajes del prácticum. Elaboración

Se dice que todo aprendizaje se produce como un proceso, esto es, algo que sucede en el tiempo, que posee una duración y que está constituido por un conjunto de fases secuenciadas que te conducen, 0 eso pretenden, al resultado final. La configuración en fases de los planes de prácticas es una condición bastante bien conocida. Todo Practicum precisa de una fase de preparación (normalmente en el propio centro universitario), una fase de desarrollo y una fase posterior de revisión de lo realizado (el plan-do-review de las pedagogías basadas en la experiencia). Cada una de dichas fases, y sobre todo la fase de desarrollo del Practicum, puede estar organizada internamente acogiendo otras subfases que mejoren su funcionalidad dentro del Plan. Muradas y Porta (2007)5 insisten en la importancia de una fase de preparación que oriente el trabajo a realizar por los estudiantes a través de consignas que los lleven a reflexionar sobre la experiencia que van a vivir. Ellas han podido demostrar que la calidad de esa reflexión está fuertemente vinculada y depende de la forma en que se conciba y desarrolle la fase de preparación del Practicum. Otro tanto podríamos decir de la capacidad de impacto sobre el Practicum que poseen tanto los modelos de supervisión habilitados (las visitas a los centros por parte

\footnotetext{
${ }^{5}$ Muradas, M. y Porta, M (2007). Las memorias del Practicum I de la titulación de Maestros de Educación Infantil: sobre qué reflexionan los alumnos, en A. Cid y otros (coords.) Buenas prácticas en el Practicum. Santiago de Compostela. Imprenta Universitaria. 977-991.
} 


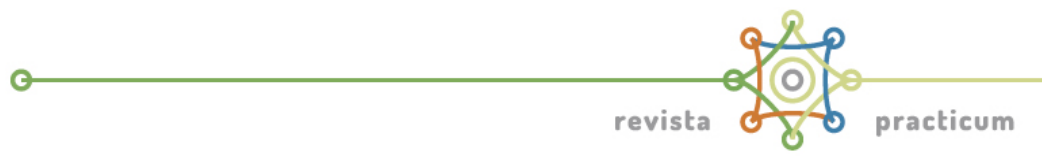

de los supervisores, las tutorías virtuales, los encuentros intermedios para revisar el desarrollo del proceso, etc.) como la naturaleza y contenidos de los productos finales que se soliciten a los alumnos en prácticas: los diarios, las memorias, los productos multimedia, los portafolios, etc. Cada uno de ellos posee virtualidades diversas y propicia diversos modos de aprendizaje y evaluación.

Un segundo aspecto importante en este apartado tiene que ver con el tipo de experiencias y/o actividades que se soliciten a los estudiantes en prácticas. Con frecuencia, estas actividades se determinan en el propio escenario de las prácticas y suelen depender más de la coyuntura institucional que de lo previsto en el propio plan de prácticas. La presión de lo cotidiano es inevitable. $Y$ no tiene por qué ser perjudicial, siempre que comporte una inmersión realista y global del estudiante en prácticas en la vida de la institución. Pero actúa en detrimento de su formación si le impide aprender bajo la supervisión de su tutor o si tal implicación lo lleva a la realización de tareas marginales o ajenas a su perfil profesional (si lo que le mandan hacer son cosas irrelevantes o superficiales desde el punto de vista de la profesión para la que se prepara; la excusa habitual de que aquí "todos tenemos que hacer de todo" vale como justificación ocasional pero no puede convertirse en una regla permanente). Aquellas prácticas en las que los alumnos van progresando en su nivel de responsabilidad, en las que poco a poco van asumiendo compromisos relevantes para el funcionamiento de la organización en la que se integran son las más interesantes y las que acaban produciendo mayor madurez en los estudiantes.

Forma parte de este mismo criterio de calidad, la necesidad de que el Practicum, en sus sucesivos periodos, vaya permitiendo a los estudiantes el hacer un recorrido representativo por las diversas secciones o modalidades de trabajo que se lleven a cabo en la empresa o institución en la que realice sus prácticas. Quienes organicen el Practicum deben estar muy atentos a esta diversificación. Algunas empresas o instituciones siguen un ritmo estacional con acciones o prioridades diversas en distintos momentos del año (las escuelas, por ejemplo), otras llevan a cabo actuaciones especializadas en sus diversos sectores o departamentos. Aunque a veces podemos plantearnos el dilema de qué sea mejor para un estudiante, si agotar su tiempo de prácticas en un solo servicio para poder conocerlo mejor o rotar por los distintos servicios 0 
departamentos, lo interesante para un alumno en prácticas, siempre que sea posible (por ejemplo, en función del tiempo disponible) es hacerse una visión lo más completa posible de las actuaciones que se llevan a cabo en el centro de prácticas. Al menos, aquellas actuaciones que tienen que ver con su perfil profesional.

En tercer lugar, el tema de la supervisión. Suele decirse que no hay buen aprendizaje sin una adecuada supervisión. Dada la complejidad de un proceso formativo que se desarrolla fuera de la institución formadora, que co-implica a diversas instituciones y personas, que se desarrolla en diversas fases y con objetivos normalmente complejos, la tarea de supervisión se hace aún más importante. Sin ella, las experiencias de Practicum corren el serio riesgo de quedarse en simples momentos de experiencias extraacadémicas, normalmente gratificantes, pero con escaso impacto en la formación de nuestros estudiantes. Por eso es tan importante la supervisión: a través de ella se ajustan los propósitos formativos del Practicum, se controlan las posibles desviaciones del sentido general del plan de prácticas, se potencia la sinergia entre los diversos implicados (especialmente entre tutores y alumnos en prácticas) y se orienta a cada estudiante en particular sobre cómo está afrontando y viviendo la experiencia.

El siguiente aspecto a considerar es la capacidad de impacto formativo del periodo de prácticas externas, esto es, la calidad de las experiencias que ha proporcionado el Practicum a nuestros estudiantes. Si se ha tratado de una experiencia bien centrada en el perfil de la profesión y funcional a los propósitos formativos encomendados al Practicum. Dos cuestiones son especialmente relevantes en este punto: si las experiencias vividas son relevantes para la profesión y si el contexto en el que se han vivido ha permitido a nuestros estudiantes aprender realmente cosas novedosas para ellos (y previsiblemente para su profesión). Vivir y experimentar algo (aunque no sea novedoso) resulta importante para el Practicum. Pero si a eso se añade la posibilidad de aprender cosas nuevas, ésa es una plusvalía relevante.

Esta cuestión suele plantear un difícil dilema a quienes coordinan y supervisan las prácticas en empresas e instituciones. Algunas empresas o instituciones son muy interesantes y acogedoras para nuestros estudiantes. En ellas tendrán oportunidad para experimentar actividades prácticas vinculadas con la profesión. Pero 
no son empresas o instituciones con un gran nivel de innovación (por el tipo de tecnología que emplean, por el tipo de actividades que desarrollan, por el tipo de personal que trabaja en ellas). Nuestros alumnos harán un buen Practicum de experimentación pero no es fácil que aprendan cosas nuevas o que tengan que enfrentarse a sistemas productivos o de servicios punteros. En el otro polo tenemos las empresas con sistemas productivos más innovadores, con profesionales de más nivel, con tecnologías de última generación. Es posible que la flexibilidad y apertura de estas empresas para acoger alumnos sea más escasa, que se corra en ellas el riesgo de que nuestros estudiantes acaben haciendo actividades marginales, etc. Pero pese a todo, conviene ser ambiciosos y perseguir modelos de Practicum que incorporen aprendizajes innovadores y de alto nivel.

Finalmente, si entendemos el Practicum como momento y situación de aprendizaje, importará mucho tomar en consideración de qué manera se ha organizado ese proceso, bajo qué enfoque de aprendizaje y priorizando qué tipo de dimensiones o propósitos formativos. En este marco, las buenas prácticas implican la incorporación de ese conjunto de elementos que se han ido consolidando como aspectos formativos clave del Practicum: la reflexión, el saber observar, el saber adaptarse a una situación nueva, la capacidad para planificar y llevar a cabo un proyecto, el conocimiento in situ de la profesión y de sus formas habituales de ser desempeñada, el conocimiento de sí mismo y de la propia adaptación a las exigencias de la profesión, etc.

El tipo de aprendizaje que se genera a través del Practicum podríamos situarlo en lo que se denomina "aprendizaje experiencial" $\left(K_{o l b}{ }^{6}\right)$ o aprendizaje a través de la experiencia que posee su propia coreografía. Se trata de aprendizajes que se realizan, por lo general, fuera del contexto convencional de las aulas y los laboratorios universitarios. Siguiendo a Oser y Baeriswyl $(2001)^{7}$ este tipo de aprendizajes posee una coreografía compleja destinada a establecer toda una red de conexiones entre lo que los sujetos van haciendo en su centro de prácticas con lo que han aprendido en las aulas y con lo que se describe en la bibliografía científica. La secuencia de fases que constituyen un proceso de aprendizaje experiencial es la siguiente:

\footnotetext{
${ }^{6}$ Kolb, D. A. (1984). Experiential Learning: Experience as the Source of Learning and Development. Prentice-Hall, Inc., Englewood Cliffs, N.J.

7 Oser, F.K. e Baeriswyl, F.J. (2001): "Choreografies of Teaching: bridging instruction to teaching", en V. RICHARDSON (Edit.): Handbook of Research on Teaching (4th Edition). Washington: AERA, páxs. 1031-1065.
} 
a) anticipación del plan de acción a desarrollar (momento en el que se establece lo que se piensa hacer, manipular, construir, resolver, etc.) y de los problemas previsibles. Es la fase de preparación del Practicum en la que se presenta el Plan de Prácticas y se consensuan con los estudiantes los aspectos que requieran acuerdo mutuo. Ya se hace normalmente. Como he señalado en un punto anterior, la actuación de los supervisores de prácticas en esta fase y las consignas que transmiten tienen una importancia fundamental en la forma en que los estudiantes afrontarán el Practicum.

b) realización de las actividades previstas en los correspondientes contextos de prácticas;

c) construcción del significado de la acción llevada a cabo a través de un intercambio comunicativo (contar lo que se ha hecho, cómo y por qué). Este es el momento en el que los estudiantes comparten su experiencia y sus valoraciones. Es un momento de aprendizaje coral que permite aprender no sólo de la propia experiencia sino, también, de la experiencia de los demás.

d) generalización de la experiencia a través de la identificación de los elementos comunes a las experiencias de los diversos sujetos. De esta manera se enriquece la experiencia y se trata de superar el nivel de experiencia parcial que cada estudiante ha podido vivir y atribuir a sus prácticas. La anécdota puede convertirse en categoría si se van uniendo las experiencias de los diversos estudiantes. Eso permitirá a cada uno de ellos hacerse una idea más global de lo que han vivido en su Practicum.

e) reflexión sobre experiencias similares existentes en la bibliografía, en los libros de texto, en bases de datos, Internet, la literatura, etc. Éste es un salto de gran magnitud. Con frecuencia, los estudiantes tienden a contraponer lo que han vivido en sus prácticas con lo que han estudiado. Lo que se está buscando es no solo evitar esa contraposición, esa ruptura entre teoría y práctica, sino justamente lo contrario, que se acostumbren a acudir a la teoría para ir resolviendo los problemas que plantea la práctica. Estudiar sobre las cuestiones problemáticas que han aparecido en el Practicum resulta muy importante.

Como puede observarse, el Practicum constituye un proceso de aprendizaje muy completo y del que los estudiantes pueden extraer grandes ventajas, incluidas las intelectuales. 


\subsection{El Practicum como experiencia personal}

Al margen de cuál sea la estructura y los propósitos formativos del Practicum, éste tiene siempre una dimensión personal que desborda ampliamente los objetivos planificables. Cada sujeto va a vivir el Practicum de una manera particular. Manera que en parte dependerá de la propia organización del periodo de prácticas y, en parte, del talante con que cada estudiante afronte y se involucre en la experiencia. Ya había recordado Gardiner $(1989)^{8}$ esa múltiple estructura formativa del Practicum señalando que las experiencias de formación deben impactar tanto en lo que él denomina aprendizajes públicos (es decir, aprendizajes pertenecientes al currículo formal) como en los aprendizajes personales (esto es, aquel tipo de mejoras que tienen que ver con el propio desarrollo personal de los estudiantes). Desde esa perspectiva, identifica tres niveles en la formación de los futuros profesionales:

1. El primer nivel se centra en lo que se ha de aprender, los contenidos (que, a veces, se convierten en resultados o metas concretas que se han de alcanzar y aparecen como requisitos para graduarse).

2. El segundo nivel se refiere al desarrollo personal y a la forma en que cada uno de ellos/as construye los significados a partir de su propia experiencia.

3. El tercer nivel se refiere al meta-aprendizaje: a cómo cada uno/a llega a identificar y hacerse consciente de su propio estilo de aprendizaje.

Es probablemente el Practicum, más que el resto de los componentes de la titulación, el momento curricular que mejor puede subvenir a la consecución de los niveles 2 y 3 de esta clasificación de Gardiner. Las particulares condiciones en que se desarrolla (fuera de la institución académica y de su cultura), las dimensiones de los sujetos que se ven envueltos en la acción (no sólo su inteligencia sino también sus emociones, sus actitudes, etc.), las diversas actividades a desarrollar (algunas de gran novedad para los estudiantes, otras de notable complejidad, otras que implican el trabajar coordinadamente

\footnotetext{
${ }^{8}$ Gardiner, D.(1989). The anatomy of supervision. Whasington, DC: Open University Press.
} 
con otras personas, etc.) hacen del Practicum un momento muy especial en la formación. De ahí su importancia en la formación personal de nuestros estudiantes.

Entre los aspectos que convendría analizar en este apartado están los siguientes:

a) la dimensión axiológica y/o social de las prácticas

b) el componente emocional de las actividades del Practicum

c) los niveles de compromiso establecidos

En primer lugar, cada vez son más, y más interesantes, las experiencias de Practicum que han tomado en consideración el componente axiológico y solidario de este momento formativo. Algunas instituciones y coordinadores de prácticas insisten en la importancia de este periodo formativo para desarrollar y poner en práctica valores y actitudes que se mencionan en los estudios pero que nuestros alumnos pocas veces tienen posibilidades de ejercer en contextos de formación (con todo lo que ello supone de estructura, supervisión, colaboración interinstitucional, etc.). En el contexto social y multicultural en el que nos ha tocado vivir, y siendo la movilidad uno de los principales mecanismos de proyección profesional que nuestros estudiantes tendrán en el futuro, esta idea va adquiriendo una importancia y un peso cada vez más fuerte en una pedagogía universitaria que quiere ir un poco más allá de los meros aprendizajes técnicos. Los modelos curriculares actuales ya suelen incluir este componente en diversos momentos de la formación (en los intercambios, en la realización de proyectos, en la oferta de materias optativas, etc.). Pero, sin duda ninguna, es el Practicum el momento privilegiado para propiciar esta dimensión formativa. Así van surgiendo los Practicum solidarios que se llevan a cabo a través de ONGs o de instituciones directamente vinculadas con el desarrollo social. En estos casos, los aprendizajes más puramente académicos pasan a un segundo plano y adquiere prioridad el hecho mismo de poder vivir y comprometerse en la ayuda a personas o grupos con necesidades específicas. Especial mención merecen, en este sentido, las numerosas experiencias de Practicum que se vienen haciendo en los últimos años en torno al "aprendizaje servicio" (Puig y otros, $2007^{9}$, Martínez ${ }^{10}$ ).

9 Puig, J. M.; Batlle, R.; Bosch, C. y Palos, J.(2007) Aprendizaje Servicio. Barcelona: Octaedro. 
En segundo lugar, el Practicum ofrece una experiencia que suele estar cargada de elementos emocionales. Este es otro de los aspectos de las prácticas que las diferencia muy claramente de las actividades académicas convencionales (las clases, los laboratorios, etc.). Los estudiantes suelen valorarlas porque, dicen, les libera por unos días de la presión de los libros y del esfuerzo más estrictamente intelectual y les sitúa en un marco de actuación en la que deben emplear todos sus recursos personales. El Practicum exige del estudiante que ponga en marcha todos sus recursos, tanto lo que sabe como lo que es. La máscara y el personaje que puede mantener durante las actividades académicas, desaparece cuando ha de enfrentarse a una situación de trabajo real y desconocida para él/ella; y hacerlo, además, en compañía de otros profesionales de los que pretende aprender. A mí me toca supervisar a estudiantes que hacen su primer periodo de Practicum. Y cada año, algunas estudiantes me comentan que no son capaces de dormir la noche anterior a iniciar sus prácticas, que se encuentran, a la vez, ansiosas e ilusionadas preguntándose si serán capaces, si se sentirán bien, si sabrán adaptarse a los niños de la clase, etc. Está claro que el Practicum les requiere otro tipo de competencias bien diversas de aquellas que han de utilizar en las clases. No me las imagino insomnes por la ansiedad e ilusión que les produce la clase que recibirán al día siguiente. Obviamente, esta condición es más atribuible a un tipo de Practicum que a otros. Los estudiantes que durante el Practicum han de verse con niños, con enfermos, con clientes de diverso tipo, con sujetos diversos a los que han de atender tendrán una especial oportunidad para auto-chequear sus características personales, sus puntos fuertes y débiles en relación con ese tipo de actuaciones profesionales.

En tercer lugar, y dentro de la misma línea de consideraciones, podemos señalar la importancia del Practicum para ir propiciando la asunción de compromisos personales, unos relacionados directamente con la profesión para la que se forman, otros vinculados a actividades genéricas de la institución en la que se realizan las prácticas. Se trata de una cualidad importante de las buenas prácticas en el Practicum: la posibilidad de que los estudiantes que lo realicen puedan-deban asumir responsabilidades (siempre relativas y supervisadas, obviamente, pues se encuentran en periodo de formación). Esos compromisos y responsabilidades irán en aumento a

${ }_{10}$ Martínez, M. (2010). Aprendizaje servicio y responsabilidad social de las universidades. Barcelona:Octaedro. 
medida que los sujetos van aproximándose al final de su formación. Pero es una de las características más importantes de un buen Practicum y la que tanto estudiantes como empleadores (o gestores del Practicum) suelen valorar más: la capacidad de comprometerse y asumir responsabilidades por parte de nuestros estudiantes. En ese sentido, hay Practicum que son fríos y burocráticos porque en ellos los estudiantes tienen escasas oportunidades de compromiso y responsabilización. Y los hay más intensos y exigentes donde, a veces desde el primer día, cada estudiante se ve enfrentado a su propia responsabilidad y ha de buscarse la vida (no sólo, obviamente) para afrontar las tareas que se le han encomendado y que dependerán de él. Aunque a veces les cuesta asumir este tipo de responsabilidades, luego vienen encantados de sus prácticas y es, justamente, una de las cosas que más valoran de ellas.

\section{En conclusión}

Aunque de forma sintética, creo que hemos podido comprobar cómo el Practicum resulta un importante componente de la formación universitaria que ofrecemos a nuestros estudiantes. Constituye, sin lugar a dudas, una mejora cualitativa que los currículos universitarios españoles han ido incorporando en los últimos años. Otros países que aún no lo han hecho, como muchos de los sudamericanos, trabajan intensamente para reformular sus propuestas curriculares y darles cabida a las prácticas. Creo que se ha extendido el convencimiento de que esa complementariedad entre la formación académica y la formación práctica en contextos profesionales reales es una condición necesaria para una buena formación. Algo que beneficia a nuestros estudiantes y hace más completa la formación que reciben.

Si tuviéramos que sintetizar, para concluir, cuáles son esas aportaciones que un buen Practicum debe proporcionar a nuestros estudiantes, podríamos indicar lo siguiente: (a) les aproxima a la cultura de la profesión para la que se forman y a las instituciones donde esa profesión se ejerce; (b) lo que han visto y experimentado durante las prácticas les ayudará a generar marcos de referencia cognitivos (decodificadores) que les permitirán entender mejor lo que aprenden en las clases y seminarios de la Universidad; (c) les ofrece experiencias formativas nuevas y complementarias a las académicas, es decir, les permite trabajar objetivos de formación que no se pueden obtener en las clases; (d) les ofrece posibilidades para el auto-conocimiento personal; (e) les ofrece mejores posibilidades de 
empleo y auto-empleo (el hecho de ver cómo funciona una empresa o un servicio puede animarles a diseñar sus propias iniciativas).

\section{Referencias bibliográficas}

Gardiner, D.(1989). The anatomy of supervision. Whasington, DC: Open University Press.

Harden, R.M. (2000). The integration ladder: a tool for curriculum planning and evaluation. Medical Education, 34(7), 551-557

Kolb, D. A. (1984). Experiential Learning: Experience as the Source of Learning and Development. Prentice-Hall, Inc., Englewood Cliffs, N.J.

Martínez, M. (2010). Aprendizaje servicio y responsabilidad social de las universidades. Barcelona:Octaedro.

Muradas, M. y Porta, M (2007). Las memorias del Practicum I de la titulación de Maestros de Educación Infantil: sobre qué reflexionan los alumnos, en A. Cid y otros (coords.) Buenas prácticas en el Practicum. Santiago de Compostela. Imprenta Universitaria. 977-991.

Oser, F.K. e Baeriswyl, F.J. (2001): "Choreografies of Teaching: bridging instruction to teaching", en V. RICHARDSON (Edit.): Handbook of Research on Teaching (4th Edition). Washington: AERA, páxs. 1031-1065.

Puig, J. M.; Batlle, R.; Bosch, C. y Palos, J.(2007) Aprendizaje Servicio. Barcelona: Octaedro.

Shulman, L.S. (1986). Paradigms and Research Programs in the Study of Teaching: a contemporary perspective, en M.C. Wittrock (Dir.), Handbook of Research on Teaching, ( $3^{a}$ ed.) (pp. 3-36), N. York: McMillan.

Zabalza, M.A. (2012). El estudio de las buenas prácticas docentes" en la enseñanza universitaria. REDU Revista de Docencia Universitaria, $10(1), 17-40$.

Zabalza, M. (2013). El Practicum y las Prácticas en Empresas en la formación universitaria. A la búsqueda de una formación equilibrada. Madrid: Narcea. 